\title{
Who are the proteolytic culprits in vascular disease?
}

\author{
William C. Parks \\ Departments of Pediatrics (Allergy and Pulmonary Medicine) and Cell Biology and Physiology, \\ Washington University School of Medicine, St. Louis, Missouri 63110, USA. \\ Phone: (314) 454-7543; Fax: (314) 454-5372; E-mail: parks_w@kids.wustl.edu
}

The extracellular matrix of the medial and adventitial layers of arteries serves several functions essential for vessel homeostasis. Beyond being an adhesive substrate for resident endothelial and smooth muscle cells and fibroblasts, vascular wall matrix proteins, such as elastin and collagen types I and III, provide resiliency, strength, and structural integrity to a tissue that is continually subjected to constant pulsatile pressure. Key to the structural function of these matrix proteins is their organization into large bundles of polymeric fibers welded together by covalent crosslinks. In healthy tissue, matrix proteins, especially fibrillar proteins, are quite stable and turn over slowly, if at all. In contrast, excessive degradation of vascular wall matrix is a key causative process in the progressive dilation of arteries during aneurysm formation (1) and in the breakdown, weakening, and eventual rupture of atherosclerotic lesions $(2,3)$.

Although a variety of proteinases, mostly released by infiltrating macrophages, have been implicated in the destruction of vessel wall matrix, the actual enzymatic culprits have not yet been conclusively fingered. Naming the guilty proteinase or proteinases is not straightforward for two main reasons. First, vascular lesions, or any inflamed tissue, are filled with many potentially destructive, extracellular proteinases of diverse gene families, principally metallo-, serine, and cysteine proteinases. Second, and more important, the actual in vivo function and scope of functions of most extracellular proteinases are not known. It is likely that many so-called matrix-degrading enzymes actually serve other functions, such as processing secreted precursor proteins, in a tissue environment. Thus, we cannot yet conclude if a specific proteinase in an inflammatory or injury setting is contributing to a reparative or disease process. Compounding this problem is a lack of animal models that fully duplicate human vascular disease. Although neointimal lesions form in injured and apolipoprotein E-deficient mice, these do not progress to rupture, the often fatal event that is generally thought to be a consequence of matrix breakdown.

Of the various proteinases present in vascular diseases, members of the matrix metalloproteinase family have received much attention, and several investigators, including myself, have proposed that these are the enzymes tearing down critical protein structures. Indeed, blocking metalloproteinase activity by treating animals with hydroxamate or tetracyclinebased inhibitors or by over-expressing TIMPs (tissue inhibitors of metalloproteinases) slows or prevents aortic dilation in elastase-induced models of aneurysm formation (4-7). Inhibiting metalloproteinase activity by similar strategies also reduces neointimal formation in injury models of restenosis and in animal models of atherosclerosis, such as apolipoprotein E-deficient mice (8-11). The inhibition seen in the restenosis and atherosclerosis models implicate metalloproteinases in the development of neointimal lesions rather than in the weakening and rupture of the vessel wall.

Serine proteinases, mostly plasminogen and its activators, u-PA and t-PA, also have a role in vascular pathology, but they seemingly function indirectly by activating the latent forms of certain prometalloproteinases, which, in turn, degrade matrix. This concept was demonstrated in a series of convincing studies by Carmeliet, Collen, and coworkers $(12,13)$. For this work, they injured arteries in Plasminogen and $u-P A$ knock-out mice or crossed these animals with apolipoprotein E-deficient mice, which, in addition to neointimal formation, apparently develop destruction of their aortic media. In both models, they found that reduced activity of some metalloproteinases correlated with protection from neointimal formation and from breakdown of medial lamellar matrix. Because elastin is the main matrix component of aortic lamellae and because it is not degraded by plasmin or $\mathrm{u}-\mathrm{PA}$, these findings suggest that metalloproteinases are destructive, at least in these mouse models of vascular disease. However, the mechanisms in human disease appear to be more complex.

In this issue of the JCI, Shi et al. (14) report that cystatin $C$, the most abundant extracellular inhibitor of cysteine proteinases, is markedly reduced in human atherosclerotic and aneurysmal lesions. Furthermore, they find that the circulating levels of cystatin $\mathrm{C}$ are significantly lower in patients with dilated abdominal aortas, as determined by ultrasonagraphy, compared with the levels in patients with a normal range of aortic diameter. Together with previous observations from this same group showing that cathepsins $\mathrm{S}$ and $\mathrm{K}$ are expressed in human vascular disease (15), these findings implicate cysteine proteinases in the pathogenesis of human vascular disease.

Several characteristics of cysteine proteinases make them intriguing suspects for causing matrix destruction in vascular disease. Although they were long thought to be strictly lysosomal enzymes that could not function in the extracellular space, many cysteine proteinase are now known to be secreted from cells, including macrophages (16), and to be active at neutral $\mathrm{pH}(17,18)$. In addition, cysteine proteinases, such as the two enzymes found in diseased arteries, efficiently degrade extracellular matrix proteins, including elastin and fibrillar collagens. Cathepsin $\mathrm{S}$ is one of the most, if not the most potent elastase known (19), and cathepsin K cleaves the triple helix of collagens I and III $(20,21)$, an activity previously thought to be restricted to collagenases of the metalloproteinase family. Inherited deficiency of cathepsin $\mathrm{K}$ in humans - and targeted deletion of its mouse homolog - results in the sclerotic bone abnormality osteopetrosis 
$(22,23)$, indicating that this proteinase is indeed important in collagen turnover. Furthermore, procathepsin L, a cysteine proteinase secreted by macrophages, gains catalytic activity against matrix proteins when it is bound to proteoglycans (24), including chondroitin sulfate proteoglycans, which are abundant in the media and in atherosclerotic lesions. Thus, because they are produced and released by infiltrating cells, are potent matrix-degrading enzymes, and, quite important, may have unchecked catalytic activity due to reduced cystatin $\mathrm{C}$ levels, cysteine proteinases may end up being the true culprits of vascular matrix destruction in aneurysms and atherosclerosis.

Although there are compelling data to implicate cysteine proteinases in matrix destruction, the observations implicating metalloproteinases are equally compelling. It is quite obvious that no single enzyme or class of enzyme will be found to be the cause of tissue destruction in aneurysms, atherosclerosis, or other conditions. Rather, as has been suggested by others, several diverse proteinases may act cooperatively to effectively degrade structural proteins. Thus, blocking the activity of one class of proteinases may significantly reduce total matrix destruction, as was suggested by the metalloproteinase inhibition studies. The new findings by Shi et al. point to an additional class of proteinases that can be targeted for intervention in vascular disease.
1. Halloran, B.G., and Baxter, B.T. 1995. Pathogenesis of aneurysms. Semin. Vasc. Surg. 8:85-92.

2. Libby, P., et al. 1996. Macrophages and atherosclerotic plaque stability. Curr. Opin. Lipidol. 7:330-335.

3. Libby, P. 1995. Molecular bases of the acute coronary syndromes. Circulation. 91:2844-2850.

4. Allaire, E., Forough, R, Clowes, M., Starcher, B., and Clowes, A.W. 1998. Local overexpression of TIMP1 prevents aortic aneurysm degeneration and rupture in a rat model. J. Clin. Invest. 102:1413-1420.

5. Curci, J.A., Petrinec, D., Liao, S., Golub, L.M., and Thompson, R.W. 1998. Pharmacologic suppression of experimental abdominal aortic aneurysms: a comparison of doxycycline and four chemically modified tetracyclines. J. Vasc. Surg. 28:1082-1083.

6. Bigatel, D.A., et al. 1999. The matrix metalloproteinase inhibitor BB-94 limits expansion of experimental abdominal aortic aneurysms. J. Vasc. Surg. 29:130-138

7. Moore, G., et al. 1999. Suppression of experimental abdominal aortic aneurysms by systemic treatment with a hydroxamate-based matrix metalloproteinase inhibitor (RS 132908). J. Vasc. Surg. 29:522-532.

8. Bendeck, M.P., Irvin, C., and Reidy, M.A. 1996. Inhibition of matrix metalloproteinase activity inhibits smooth muscle cell migration but not neointimal thickening after arterial injury. Circ. Res. 78:38-43.

9. George, S.J., Johnson, J.L., Angelini, G.D., Newby, A.C., and Baker, A.H. 1998. Adenovirus-mediated gene transfer of the human TIMP-1 gene inhibits smooth muscle cell migration and neointimal formation in human saphenous vein. Hum. Gene Ther. 9:867-877.

10. George, S.J., Baker, A.H., Angelini, G.D., and Newby, A.C. 1998. Gene transfer of tissue inhibitor of metalloproteinase- 2 inhibits metalloproteinase activity and neointima formation in human saphenous veins. Gene Ther. 5:1552-1560.

11. Rouis, M., et al. 1999. Adenovirus-mediated overexpression of tissue inhibitor of metalloproteinase-1 reduces atherosclerotic lesions in apolipoprotein E-deficient mice. Circulation 100:533-540.

12. Carmeliet, P., et al. 1997. Urokinase-generated plasmin activates matrix metalloproteinases during aneurysm formation. Nat. Genet. 17:439-444.
13. Lijnen, H.R., et al. 1998. Function of the plasminogen/plasmin and matrix metalloproteinase systems after vascular injury in mice with targeted inactivation of fibrinolytic system genes. Arterioscler. Thromb. Vasc. Biol. 18:1035-1045.

14. Shi, G.-P., et al. 1999. Cystatin C deficiency in human atherosclerosis and aortic aneurysms. $J$. Clin. Invest. 104:1191-1197.

15. Sukhova, G.K., Shi, G.-P., Simon, D.I., Chapman, H.A., and Libby, P. 1998. Expression of elastolytic cathepsins $\mathrm{S}$ and $\mathrm{K}$ in human atheroma and regualtion of their production in smooth muscle cells. J. Clin. Invest. 102:576-583.

16. Reddy, V.Y., Zhang, Q.Y., and Weiss, S.J. 1995. Pericellular mobilization of the tissue-destructive cysteine proteinases, cathespins $\mathrm{B}, \mathrm{L}$, and $\mathrm{S}$, by human monocyte-derived macrophages. Proc. Natl. Acad. Sci. USA. 92:3849-3853.

17. Brömme, D., et al. 1993. Functional expression of human cathepsin $\mathrm{S}$ in Saccharomyces cerevisiae. Purification and characterization of the recom binant enzyme. J. Biol. Chem. 268:4832-4838.

18. Dehrmann, F.M., Coetzer, T.H., Pike, R.N., and Dennison, C. 1995. Mature cathepsin L is substantially active in the ionic milieu of the extracellular medium. Arch. Biochem. Biophys. 324:93-98.

19. Shi, G.-P., Munger, J.S., Meara, J.P., Rich, D.H., and Chapman, H.A. 1992. Molecular cloning and expression of human alveolar macrophage cathespin S, an elastolytic cysteine proteinase. J. Biol. Chem. 267:7258-7262.

20. Garnero, P., et al. 1998. The collagenolytic activity of cathepsin $\mathrm{K}$ is unique among mammalian proteinases. J. Biol. Chem. 273:32347-32352.

21. Kafienah, W., Bromme, D., Buttle, D.J., Croucher, L.J., and Hollander, A.P. 1998. Human cathepsin $\mathrm{K}$ cleaves native type I and II collagens at the $\mathrm{N}$ terminal end of the triple helix. Biochem. J. 331:727-732.

22. Gelb, B.D., Shi, G.-P., Chapman, H.A., and Desnick, R.J. 1996. Pyconodysostosis, a lysosomal disease caused by cathepsin K deficiency. Science. 273:1236-1238

23. Saftig, P., et al. 1998. Impaired osteoclastic bone resorption leads to osteopetrosis in cathespin K-deficient mice. Proc. Natl. Acad. Sci. USA. 95:13452-13458.

24. Ishidoh, K., and Kominami, E. 1995. Procathep$\sin \mathrm{L}$ degrades extracellular matrix proteins in the presence of glycosaminoglycans in vitro. Biochem. Biophys. Res. Commun. 217:624-631. 